\title{
Brucellar arthritis in children and its successful treatment with trimethoprim-sulphamethoxazole (co-trimoxazole)
}

FRANCISCO J GÓMEZ-REINO, ISABEL MATEO, ANTONIO FUERTES, ANDP̣ JUAN J GOMEZ-REINO

From the Rheumatology Service, Paediatric Rheumatology Unit, and Microbiology Service, Hospital $1^{0-}$ de Octubre, Madrid, Spain

SUMMARY The clinical characteristics and the response to treatment with high doses of co-trimoxazole in 12 children with brucellar arthritis were analysed retrospectively. The childreno lived in an urban area and all but two had a history of unprocessed milk or cheese ingestion. $\rightarrow$ Fever and oligoarthritis of the lower extremities were the most common clinical findings. Controls of the disease was achieved by three months of treatment. Compliance with the medication was excellent, and no significant side effects occurred.

Key phrases: chronic infection of the joints, treatment of joint infection, co-trimoxazole therapy.

Human brucellosis is still found in European countries where pasteurisation of milk is commonplace but illegally marketed dairy products are also available. Between 9 and $20 \%$ of cases occur in children, and about one third of them develop arthritis. ${ }^{1}$ Treatment includes the long term use of tetracycline and streptomycin. In children this form of therapy is limited by side effects and toxicity. Recently, successful control of the disease in adults with co-trimoxazole has been reported. ${ }^{2} 3$ Over a four year period we have seen 12 children with brucellar arthritis and have treated them with high doses of co-trimoxazole. In this report we describe our experiences with this group of patients.

\section{Patients and methods}

We reviewed the records of 12 children with brucellar arthritis attending our Paediatric Rheumatology Unit between January 1979 and December 1982. Particular attention was paid to a previous history of ingestion of unprocessed dairy products or contact with cattle, presenting features, musculoskeletal symptoms and signs, and the response to therapy. Laboratory tests reviewed included full

Accepted for publication 13 August 1985.

Correspondence to Dr Isabel Mateo, Servicio de Reumatologia, Hospital 10 de Octubre, Carretera de Andalucia Km 5,4, Madrid 28041, Spain. blood counts, urinalysis, SMAC (Thecnicón), anda serological tests and blood cultures for brucella.

\section{Results}

The mean age of the children was 9.5 years (range 3-14), with an equal sex distribution (male/female 5/7). All but one lived in an urban area. Of the $12 \stackrel{\frac{0}{0}}{2}$ eight had previously ingested unprocessed milk oro cottage cheese (one also had contact with cattle) $\underline{3}$. Two did not have clear exposure to contaminated food or animals, and no relevant information wase available about the remaining two.

The interval between onset of symptoms and diagnosis ranged from five days to two years.․․ Patients with hip involvement had the longest delay ( $>$ five months). Systemic signs and symptoms wereo absent except for fever $\left(>38.5^{\circ} \mathrm{C}\right)$ in seven patients. 0 All had arthritis and six had arthralgias related to non-inflamed joints (Table 1). The arthritis waso characterised mostly by soft tissue swelling without obvious effusions. A total of 21 joints were inflamed (Table 1). Hips, elbows, and knees were ofteno? involved, while small joints of the hands, feet, and spine were never affected.

Routine laboratory investigations were unremark- $-\vec{\Phi}$ able. Eleven patients had normal blood counts and one eosinophilia. The erythrocyte sedimentation? rate was raised $(>20 \mathrm{~mm} / 1 \mathrm{st} \mathrm{h})$ in six patients. 
Table 1 Joints involved in 12 children with brucellar arthritis

\begin{tabular}{|c|c|c|c|c|}
\hline \multirow[t]{2}{*}{ Joint } & \multicolumn{2}{|l|}{$\begin{array}{l}\text { Arthritis* } \\
n=21 \quad(\%)\end{array}$} & \multicolumn{2}{|c|}{$\begin{array}{l}\text { Arthralgia* } \\
n=13(\%)\end{array}$} \\
\hline & Unilateral & Bilateral & Unilateral & Bilateral \\
\hline Hip & $2 \quad(9 \cdot 5)$ & $4(38 \cdot 1)$ & $2(15 \cdot 4)$ & - \\
\hline Elbow & $4(19 \cdot 0)$ & - & $1(7 \cdot 7)$ & - \\
\hline Knee & $3(14 \cdot 3)$ & - & $2(15 \cdot 4)$ & $3(46 \cdot 2)$ \\
\hline Ankle & $1 \quad(4 \cdot 8)$ & $1 \quad(9 \cdot 5)$ & - & - \\
\hline Sacroiliac & $1 \quad(4 \cdot 8)$ & - & - & - \\
\hline Shoulder & - & - & - & $1(15 \cdot 4)$ \\
\hline
\end{tabular}

${ }^{*}$ See text for description.

Biochemical parameters were normal in all but two, who had moderate increases of serum transaminases. Brucella melitensis was isolated from the blood of four of the 12 patients. Serological tests were always positive (Table 2 ).

Radiographic studies showed narrowing of the joint space in two hips and one sacroiliac joint, juxta-articular osteopenia in one elbow, and soft tissue swelling in the remaining joints.

All patients were treated orally for 12 weeks with co-trimoxazole (Table 3). Eleven were followed up for a minimum of two years and one was lost to follow up after six months. The therapeutic response was good, the arthritis subsiding within two to three months in all. Two patients with hip involvement, however, still have functional limitation of the joint. Relapses of the disease were not seen. Compliance with the medication was excellent, and no side effects were seen or reported.

Table 2 Serology and blood culture results in 12 children with brucellar arthritis

\begin{tabular}{llrl}
\hline Patient No & Blood culture & ATT & CMT \\
\cline { 1 - 2 } 1 & Positive & 320 & 1280 \\
2 & Positive & 2560 & 2560 \\
3 & Positive & 160 & ND $\ddagger$ \\
4 & Positive & 320 & ND \\
5 & Negative & 80 & 1260 \\
6 & Negative & 160 & 320 \\
7 & Negative & 320 & 640 \\
8 & Negative & 40 & 320 \\
9 & Negative & 160 & 640 \\
10 & Negative & 640 & 1280 \\
11 & Negative & 160 & 1280 \\
12 & Negative & 640 & 1280 \\
\hline
\end{tabular}

${ }^{*}$ ATT $=$ agglutination test tube $\left(\right.$ dilution $\left.^{-1}\right)$. $+\mathrm{CMT}=$ Coombs' microtitre test $\left(\right.$ dilution $\left.^{-1}\right){ }^{\text {. }}$ $\ddagger \mathrm{ND}=$ not done.
Table 3 Co-trimoxazole dosage* used for the treatment of brucellar arthritis in children

\begin{tabular}{ll}
\hline Dose $(\mathrm{mg} / \mathrm{kg}$ body wt/24 $h)$ & Week \\
\hline $90-100$ & 1 \\
$50-60$ & $2-4$ \\
$25-30$ & $5-12$
\end{tabular}

${ }^{*}$ Dosage refers to sulphamethoxazole because it was used in the standard fixed combination form.

\section{Discussion}

Brucellosis should be included in the differential diagnosis of children with oligoarthritis and fever in localities where this infection still occurs. ${ }^{4}$ Several features of the disease merit consideration. First, in our own and in previous experience ${ }^{1}$ the absence of recognised exposure to potentially contaminated food or animals does not exclude the diagnosis. Secondly, neither clinical nor routine laboratory abnormalities are distinctive. Awareness is thus important because diagnosis depends on cultural or serological evidence, or both. Thirdly, in contrast with adults, ${ }^{1}$ children seldom have axial skeleton involvement.

The efficacy and safety of high doses of cotrimoxazole for treatment of brucellosis in children has not previously been studied. In this regard it might be argued that despite a full course of treatment two of our patients have residual functional hip limitation. However, this can occur in other hip infections despite effective chemotherapy. ${ }^{5}$ Could spontaneous remissions and relapses of the disease ${ }^{1}$ have accounted for some of the response? If this were the case we should have seen relapses during the two years of observation. Finally, it should be noted that our patients do not represent the whole spectrum of this disease. Children were referred to our Unit because of arthritis, which is present in only one third of infected patients. Toxicity could limit the treatment with certain drugs. The dose of co-trimoxazole used was higher than that recommended for urinary tract infections, otitis media, and shigellosis, ${ }^{6}$ though similar to the one used in the treatment of Pneumocystis carinii infections. ${ }^{7}$ Despite this high dose and the length of treatment we did not encounter significant side effects.

\section{References}

1 Gotuzzo E, Alarcon J S, Bocanegra T S, et al. Articular involvement in human brucellosis: a retrospective analysis of 304 cases. Semin Arthritis Rheum 1982: 245-55. 
2 Velasco A C, Gutierrez-Altes A, Rodriguez Noriega A. Tratamiento de la brucellosis con sulfametoxazol-trimetoprim: evaluación de los resultados en 40 pacientes. In: Baquero F, ed. Proceedings of the Mediterranean Congress of Chemotherapy. Madrid, 1978: 765-8.

3 Kontoyannis P A, Papapoulos S E. Mortoglou A A. Cotrimoxazole in chronic brucellosis: a two year follow up. $\mathrm{Br}$ Med $J$ 1975; ii: 480-1.

4 Walter L Norton. Brucellosis and rheumatic syndromes in Saudi Arabia. Ann Rheum Dis 1984; 43: 810-5.
5 Morrey B F, Bianco A J, Rhodes K H. Suppurative arthritis ow the hip in children. J Bone Joint Surg 1976: 58A: 388-92. 6 Wormser G P. Keusch G T. Trimethoprim-sulfamethoxazole irş the United States. Ann Intern Med 1979; 91: 420-9.

7 Hughes W I. Kuhn S. Chaudhary S, et al. Successful chemo prophylaxis for Pneumocystis carinii pneumonitis. N Engl J Mec 1977: 297: 1419-26.

8 Otero J R, Fuertes A, Palenque E, et al. Microtiter adaptes $\mathbb{B}$ method that facilitates the Coombs' test for brucellosis. J Clin bu Microbiol 1982; 16: 737-8. 